\title{
The Possible Way to Evaluate the Purity of Double Walled Carbon Nanotubes over Single Wall Carbon Nanotubes by Chemical Doping
}

Yoong-Ahm Kim, Hiroyuki Muramatsu, Masahito Kojima, Takuya Hayashi and Morinobu Endo Faculty of Engineering, Shinshu University 4-17-1 Wakasato, Nagano-shi 380-8553, Japan

Mauricio Terrones

Advanced Materials Department, IPICYT, Camino a la Presa San José 2055, Col. Lomas $4^{\mathrm{a}}$. Sección, San Luis Potosí 78216, México

Mildred S. Dresselhaus

Massachusetts Institute of Technology, Cambridge, Massachusetts 02139-4307, USA

\begin{abstract}
Here we carried out Raman study on chemically doped single wall carbon nanotube (SWNT)/double wall carbon nanotube (DWNT) mixed bucky-papers. Their highly different Raman responses (e.g., a large up-shift of tangential mode of SWNT and no large changes in the frequencies of tangential mode assigned to the outer tubes of the DWNT) upon doping with the sulfuric acid could be used as a qualitative indicator of the purity of the DWNT samples with the concentration of its SWNTs contents.

*Corresponding author: Tel: +81-26-269-5212, Fax: +81-26-269-5208, E-mail: yak@endomoribu.shinshu-u.ac.jp
\end{abstract}

\section{Introduction}

Double-walled carbon nanotubes (DWNTs), with a co-axial structure, that occupies a position between multi-walled carbon nanotubes (MWNTs) and single wall carbon nanotubes (SWNTs), have attracted much attention by numerous scientists because they are expected to exhibit interesting structural and electronic properties [1]. In this sense, various synthetic methods to produce DWNTs have been published; arc discharge [2], chemical vapor deposition [3-5] and thermal treatment of fullerene peapods [6, 7]. This latter method usually generates mixtures of DWNTs and SWNTs because the incomplete filling of fullerenes in the core of the SWNTs results in shortened inner tubes. Another reason for growing a mixture of DWNTs and SWNTs is that the catalytic growth conditions of DWNTs have proved to be very similar in comparison to those of SWNTs, including the same distribution of metal particles [8]. However, the 
fabrication of highly pure DWNT material is needed in order to probe the electronic as well as the transport and mechanical properties of individual DWNTs. Recently we reported the fabrication of highly purified DWNTs [9] through the right combination of a CVD method [10, 11] with an optimized two-step purification process. Even though a detailed high resolution transmission electron microscopy (HR-TEM) study on samples only gives us some information about the purity of carbon nanotubes, such as the ratio of DWNTs to SWNTs, the intrinsic drawback of this technique is that data are recorded only for a limited amount of sample, like in the case of the scanning electron microscope (SEM) [12]. In this context, the development of a simple technique for evaluating the purity of DWNTs relative to the portion of SWNTs in a bulk sample is very much needed.

We report here, for the first time, a very simple method to evaluate the purity of a DWNT sample regarding the presence of SWNTs based on a Raman study on chemically doped SWNT and DWNT mixtures. Raman spectroscopy has been widely utilized for studying both structural information (e.g., tube diameter and the degree of structural perfection) of SWNTs [13] and DWNTs [6, 14-15] and information on the alteration of the electronic structure of SWNTs [16, 17] and DWNTs $[18,19]$ produced by chemical dopants. Highly different Raman sensitivities of SWNTs and DWNTs to chemical dopants (e.g., concentrated $\mathrm{H}_{2} \mathrm{SO}_{4}$ ) give us some clue to evaluate the purity of a DWNT sample with regard to its concentration of SWNTs.

\section{Experimental}

Highly purified arc-derived SWNTs purchased from the Iljin Company (Korea) were used without further purification (see SEM and TEM images of SWNTs in Fig. 1 (a)-(c)). Highly purified DWNTs were obtained by a CVD method and an optimized purification process, as previously described (see SEM and TEM images of DWNTs in Fig. 1 (d)-(f) [9]. For observing a doping effect in the Raman spectra, a series of seven SWNT and DWNT mixtures $($ SWNT/DWNT (weight ratio) $=10 / 0,8 / 2,6.6 / 3.3,5 / 5,3.3 / 6.6,2 / 8$ and 0/10) were prepared. The two types of carbon nanotubes were homogeneously dispersed in concentrated nitric acid for 5 hours with the help of ultra-sonication. It should be note that both samples have a large-sized bundle in the range form 20 to 50nm. Thus, we were able to disperse both samples only by strong nitric acid treatment, and also confirmed that there was no structural damage through this acid treatment. Then, by filtering a stable suspension of SWNT and DWNT mixtures (polytetrafluoroethylene (PTFE) filter, $1 \mu \mathrm{m}$ ), rinsing the resulting material with de-ionized water several times, drying it for $24 \mathrm{hrs}$ in vacuum and peeling off the paper from the PTFE filter, we obtained thin and flexible black-colored papers. Finally, a series of black-papers were dipped in concentrated sulfuric acid (97\%) for a short time. No large changes in the D 
band spectra via chemical doping were observed, strongly suggesting that sulfuric acid treatment did not induce structural damage to the series of SWNT/DWNT samples. For a series of chemically doped and undoped samples, we obtained Raman spectra using the Ni:Yag laser line at 532nm $(2.33 \mathrm{eV})$ in the back-scattering geometry using a Kaiser HoloLab5000 system.

\section{Results and discussion}

Figure 2 shows the low-frequency radial breathing mode (RBM) spectra for a series of seven SWNT/DWNT mixtures. The RBM Raman features corresponding to the coherent vibration of the $\mathrm{C}$ atoms in the radial direction have been utilized to study the nanotube diameter through the RBM frequency. Using the equation $\omega_{R B M}=218.3 / d_{t}+15.9$ [9], where $d_{t}$ is the tube diameter (nm) and $\omega_{R B M}$, the RBM frequency $\left(\mathrm{cm}^{-1}\right)$, it is possible to obtain the tube diameters of SWNTs (ca. $1.40 \mathrm{~nm}$ ) and DWNTs (inner:outer diameters $=0.74 \mathrm{~nm}: 1.41 \mathrm{~nm}$ and $0.87 \mathrm{~nm}: 1.56 \mathrm{~nm}$ ), consistent with detailed HR-TEM observations. It is noteworthy that the outer diameter of the DWNTs is very similar to that of the SWNTs. With an increasing fraction of DWNTs, the relative RBM intensity, assigned to the inner tubes of the DWNTs over that of the outer tubes of the DWNTs and SWNTs, increases monotonically. In the case of the doped SWNTs, an upshift in the RBM frequency (ca. $7 \mathrm{~cm}^{-1}$ ) [16] is observed upon doping with $\mathrm{H}_{2} \mathrm{SO}_{4}$. On the other hand, no large change is observed in the spectra for the doped DWNTs, except for a reduced RBM intensity assigned to the outer tube, indicating that the dopant only reached the outer tubes of the DWNTs. Even though we could not probe the purity of a DWNT sample with regard to their SWNT content from RBM features for the doped SWNTs and DWNTs mixed samples, we can see the presence of DWNTs over SWNTs because no large change is expected upon chemical doping because the intensity and the frequency of RBM of the inner tube completely protected by the outer tube of the DWNTs.

The tangential mode (TM) frequency has been used to probe the charge transfer between dopant and SWNTs or DWNTs (e.g., upshift of G band for acceptors) [16-19]. As shown in Fig. 3, a large up-shift of the TM (ca. $16 \mathrm{~cm}^{-1}$ ) in the case of doped SWNTs can be clearly explained by charge-transfer because sulfuric acid is known to be a strong acceptor in graphite intercalation compounds [20]. On the other hand, the result of doped DWNTs is quite different. In terms of the tight-binding model, the incident photon $(532 \mathrm{~nm})$ can resonate with the $\mathrm{E}_{33}{ }^{\mathrm{S}}$ (outer) and $\mathrm{E}_{11}{ }^{\mathrm{M}}$ (inner) transitions, where $E_{i i}$ is the energy difference between the Van Hove singularities between filled and empty states [13]. The frequency of the TM at $1572 \mathrm{~cm}^{-1}$ assigned to the inner tubes was slightly up-shifted (ca. $9 \mathrm{~cm}^{-1}$ ) upon doping with $\mathrm{H}_{2} \mathrm{SO}_{4}$, possibly due to the partial charge transfer (ca. 10\%) from the outer tubes to the inner tubes [18]. It is interesting to note 
that the intensity of the TM assigned to the outer tubes $\left(1592 \mathrm{~cm}^{-1}\right)$ is highly depressed without any changes of frequency. One possible explanation for this phenomenon was suggested as the loss of Raman resonance through the modified optical transitions upon chemical doping [21]. Upon doping with $\mathrm{H}_{2} \mathrm{SO}_{4}$ the charge transfer between dopant and the outer tube of the DWNT resulted in a loss of Raman resonance of TM assigned to the outer tubes, due to a depleting of the optical transition between the van Hove singularities of the DWNTs, and as a result, the incident phonon only resonate with the remained inner tubes $\left(\mathrm{E}_{11}{ }^{\mathrm{M}}\right)$. Resultantly, largely different Raman responses between the $\mathrm{G}^{+}$of SWNT and the TM of the outer tube of the DWNTs upon chemical doping could show us how to evaluate qualitatively the purity of DWNTs samples with regard to the small amount of SWNTs they contain. For samples containing a high fraction of SWNTs (> 50\%), we can easily evaluate the purity of samples by measuring the up-shift of the TMs (ca $\left.16 \mathrm{~cm}^{-1}\right)$. On the other hand, we have to be careful when evaluating the purity of DWNT samples with regard to a low fraction of SWNTs (<50\%) (e.g., the SWNT:DWNT = 2/8 and 3.3/6.6 mixed samples). For example, if we used the present DWNT sample as a standard sample for evaluating the purity of DWNTs over SWNTs (practically, it is impossible to obtain a fully $100 \%$ DWNT sample), the monotonic changes of shoulder lines (around $1605 \mathrm{~cm}^{-1}$ ) by chemical doping (see short arrows in Fig. 3 (a)) with decreasing amounts of SWNTs could be used for a qualitative purity evaluation of DWNTs with regard to the minor fraction of SWNTs contained in this sample. To understand the changes of TM profiles in detail, curve fitting was carried out for three samples were carried out (see Fig. 3 (b) and (c)). We can fit the pristine and doped TM of DWNT by three Lorentzians, where the strongest TM originates from the outer tube whereas the shoulder peak at around $1577 \mathrm{~cm}^{-1}$ from the inner tube of the DWNT. For samples (e.g., SWNT/DWNT $=2 / 8$ and 3.3/6/6), four Lorentzians were well fitted because the broadened bumps at around $1606 \mathrm{~cm}^{-1}$ is believed to result from the highly up shifted $\mathrm{G}^{+}$line of the SWNT. Thus, at least we can notice the presence of SWNTs by careful analysis of TM profiles for the chemically doped DWNT sample.

We also observed a single dominant Raman feature at $2677 \mathrm{~cm}^{-1}$ (G' band) in the high-order Raman spectra of SWNTs, which is a highly symmetric line (see Fig. 4 (a)). With increasing fraction of DWNTs, the intensity of the newly developed Raman lines at ca. $2630 \mathrm{~cm}^{-1}$ increase monotonically. M. Kalbac et al. [22] assigned the Raman line at ca. $2630 \mathrm{~cm}^{-1}$ to the inner tubes and the Raman line at $2677 \mathrm{~cm}^{-1}$ to the outer tubes by observing Raman spectra in peapod-derived DWNTs. It is noteworthy that the shapes of the G'-bands were varied upon the concentration of DWNTs linearly and furthermore clearly split into two peaks for all samples containing DWNTs by the chemical doping. In detail, in order to characterize the change of the G'-band with the fraction of DWNTs, we carried out the curve fitting of the G' band using Lorentzian lineshape functions. The Raman frequency assigned to the outer tubes is up-shifted 
whereas Raman frequency assigned to the inner tubes is down-shifted through chemical doping with $\mathrm{H}_{2} \mathrm{SO}_{4}$ (see Fig. 4 (b)). The differences in Raman frequencies assigned to the outer tubes via chemical doping were noticeable for a sample containing a large fraction of SWNTs. In addition, a relatively linear relation between the fraction of DWNTs and the ratio of $I_{G^{\prime}(-)} / I_{G^{\prime}(+)}$ could be used as a qualitative indicator for evaluating the purity of DWNT sample with regard to their content of SWNTs (Fig. 4 (c)).

\section{Conclusion}

In summary, we carried out the chemical doping of a series of seven SWNT/DWNT mixtures with a typical acceptor intercalant (e.g., $\mathrm{H}_{2} \mathrm{SO}_{4}$ ). A relatively large up-shift of the TM in SWNTs was observed, in close agreement with the result of ref [16]. Whereas no changes in the frequency of the TM spectra assigned to the outer tubes in DWNTs was observed, a greatly decreased intensity was seen for the outer tubes both for the TM and RBM features upon doping with $\mathrm{H}_{2} \mathrm{SO}_{4}$, even though the diameter of the SWNTs is very similar to the outer diameter of the DWNTs. The quite different TM and G' band responses of SWNTs and DWNTs to chemical doping could be used as a qualitative indicator of the purity of the DWNT samples regarding their concentration of SWNTs. Unfortunately, we were only able to use the resonance Raman tool for evaluating the purity of DWNTs or SWNTs through chemical doping for carbon nanotubes with relatively large diameters (above $2 \mathrm{~nm}$ ) due to notch filter limitations (which restrict observations of the $\omega_{R B M}$ to be made only above $100 \mathrm{~cm}^{-1}$ ). It is clear that a small fraction of SWNTs in a DWNT sample allows observation of an enormous effect in the Raman spectra, especially for chemically doped samples. This preliminary study will be utilized for evaluating the purity of a DWNT sample with the concentration of its SWNTs through multi-laser Raman studies.

\section{Acknowledgements}

This work was supported by the CLUSTER of Ministry of Education, Culture, Sports, Science and Technology. MSD acknowledges the support from NSF grant DMR 04-05538. This work was also sponsored by CONACYT-Mexico (MT) and the MIT-CONACYT program (MSD, MT). 


\section{Figure captions}

Figure 1 FE-SEM (a, d), low resolution TEM (b, e) and cross-sectional high resolution TEM (c, f) images of SWNTs and DWNTs, respectively (Insets are their corresponding magnified FE-SEM images). Note that both samples exist in a bundle state.

Figure 2 Low-frequency Raman spectra of a series of undoped and doped SWNT/DWNT mixture samples. Concentrated sulfuric acid was used as a dopant. The dashed lines (blue color) indicate undoped samples whereas the dotted lines (red color) indicate doped samples, respectively.

Figure 3 (a) High-frequency TM Raman spectra of a series of undoped and doped SWNT/DWNT mixture samples. The dashed lines (blue color) indicate undoped samples whereas the dotted line (red color) indicates doped samples, respectively. Enlarged and curve fitted Raman spectra of the pristine (b) and chemically doped (c) mixed samples (e.g., SWNT/DWNT = 0/10, 2/8 and 3.3/6.6).

Figure 4 Second-order Raman spectra of a series of undoped (a) and doped (b) SWNT/DWNT mixture samples. The thin dotted lines (black color) = original data; the solid line (black color) = the fitted result of two Lorentzian; the thick dotted line (blue color) $=$ G'(-); the thick dotted line (red color) $=G^{\prime}(+)$. Variations of frequencies (c) and $I_{G^{\prime} L F} / I_{G^{\prime} H F}$ (the intensity of G' band at low frequency over the intensity of G' band at high frequency) as a function of SWNT (or DWNT) contents. 


\section{References}

[1] Saito, R.; Matsuo, R.; Kimura, T.; Dresselhaus, G.; Dresselhaus, M. S. Chem. Phys. Lett. 2001, 348, 187-193.

[2] Sugai, T.; Yoshida, H.; Shimada, T.; Okazaki, T.; Shinohara, H. Nano Lett. 2003, 3, 769-773.

[3] Flahaut, E. ; Basca, R. ; Peigney, A. ; Laurent, C. Chem. Commun. 2003, 1442- 1443.

[4] Lyu, S. C.; Liu, B. C. ; Lee, S. H. ; Park, C. Y. ; Kang, H. K. ; Yang, C. W. ;. Lee, C. J. J. Phys. Chem. B 2004, 108, 2192-2194.

[5] Wei, J.; Ci, L.; Jiang, B.; Li, Y.; Zhang, X.; Zhu, H.; Xu, C.; Wu, D. J. Mater. Chem. 2003, 13, 1340-1344.

[6] Bandow, S. ; Takizawal, M. ; Hirahara, K.; Yudasaka, M.; Iijima, S. Chem. Phys. Lett. 2001, 337, 48-54.

[7] Pfeiffer, R.; Kuzmany, H. ; Kramberger, C.; Schaman, C.; Pichler, T.; Kataura, H.; Achiba, Y. ; Kurti, J. ; Zolyomi, V. Phys. Rev. Lett. 2003, 90, 225501.

[8] Muramatsu, H.; Hayashi, T.; Kim, Y. A.; Endo, M.; Terrones, M.; Dresselhaus, M. S. J. Nanosci. Nanotechno. 2005, 5, 1-5.

[9] Endo, M.; Muramatsu, H.; Hayashi, T.; Kim, Y. A.; Terrones, M.; Dressehaus, M. S. Nature 2005, 433, 476.

[10] Oberlin, A.; Endo, M.; Koyama, T. J. Cryst. Grow. 1976, 32, 335-349.

[11] Endo, M. Chem Tech 1988, 18, 568-576.

[12] Itkis, M.E.; Perea, D. E.; Jung, R.; Niyogi, S.; Haddon, R. C. J. Am. Chem. Soc. 2005, 127, 3439-3448.

[13] Dresselhaus, Eklund, P. C. Adv. Phys. 2000, 49, 705.

[14] Bacsa, R. R.; Peigney, A.; Laurent, Ch.; Puech. P.; Bacsa, W. S. Phys. Rev. B 2002, 65, 161404.

[15] Pfeiffer, R.; Kuzumany, H.; Kramberger, Ch.; Schaman, Ch.; Pichler, T.; Kataura, H.; Achiba, Y.; Kurti, J.; Zolyomi, V. Phys. Rev. Lett. 2003, 90, 225501.

[16] Rao, A. M.; Eklund, P. C.; Bandow, S.; Thess, A.; Smalley, R.E. Nature 1997, 388, 257-259.

[17] Bendiab, N.; Righi, A.; Anglaret, E.; Sauvajol, J.-L.; Duclaux, L. ; Beguin, F. Chem. Phys. Lett. 2000, 339, 305.

[18] Chen, G.; Bandow, S.; Margine, E. R.; Nisoli, C.; Kolmogoro, A. N.; Crespi, V. H.; Gupta, R.; Sumanasekera, G. U.; Iijima, S.; Eklund, P. C. Phys. Rev. Lett. 2003, 90, 257403.

[19] Cambedouzou, J.; Sauvajol, J.-L.; Rahmani, A.; Flahaut, E.; Peigney, A.; Laurent, C. Phys. Rev. B 2004, 69, 235422. 
[20] Zabel, H.; Solin, S. A. Graphite Intercalation Compounds II-Transport and electronic properties 1992, Springer-Verlag, p. 145.

[21] P. Petit, C. Mathis, G. Journet, P. Bernier, Chem. Phys. Lett. 1999, 305, 370-374.

[22] Kalbac, M.; Kavan, L.; Zukalova, M.; Dunsch, L. Carbon 2004, 42, 2915-2920. 